\title{
Spatial and Temporal Behavior of Microwave Backscatter Directional Modulation Over the Saharan Ergs
}

David G. Long

david_long@byu.edu

Haroon Stephen

Follow this and additional works at: https://scholarsarchive.byu.edu/facpub

Part of the Electrical and Computer Engineering Commons

\section{Original Publication Citation}

Stephen, H., and D. G. Long. "Spatial and Temporal Behavior of Microwave Backscatter Directional Modulation Over the Saharan Ergs." Geoscience and Remote Sensing, IEEE Transactions on 45.5 (27): 1164-73

\section{BYU ScholarsArchive Citation}

Long, David G. and Stephen, Haroon, "Spatial and Temporal Behavior of Microwave Backscatter Directional Modulation Over the Saharan Ergs" (2007). Faculty Publications. 258.

https://scholarsarchive.byu.edu/facpub/258 


\title{
Spatial and Temporal Behavior of Microwave Backscatter Directional Modulation Over the Saharan Ergs
}

\author{
Haroon Stephen, Member, IEEE, and David G. Long, Senior Member, IEEE
}

\begin{abstract}
Radar backscatter $\left(\sigma^{\circ}\right)$ from ergs is modulated with view direction [incidence $(\theta)$ and azimuth $(\phi)$ angles], where the modulation characteristics reflect the surface geometry. $\sigma^{\circ}$ also varies spatially and reflects the spatial inhomogeneity of the sand surface. We use $\sigma^{\circ}$ measurements at different $\theta$ and $\phi$ angles from the NASA, European Remote Sensing satellite, and SeaWinds scatterometers to understand the relationship between wind and erg bedforms. A model incorporating the $\sigma^{\circ} \phi$-modulation and spatial inhomogeneity is proposed. Surface slope variations are related to the $\sigma^{\circ}$ spatial inhomogeneity. We compare the backscatter model results with numerically predicted wind direction data provided by the European Centre for Medium-Range Weather Forecasts (ECMWF) over the erg surfaces. We use the maxima of the $\phi$-modulation at $\theta=33^{\circ}$ to infer the orientation of the dominant slip-sides on the sand surface. These orientations are consistent with the ECMWF wind directions spatially and temporally.
\end{abstract}

Index Terms-Desert, dunes, ergs, European Centre for Medium-Range Weather Forecasts (ECMWF), European Remote Sensing Satellite Scatterometer (ESCAT), NASA Scatterometer (NSCAT), SeaWinds Scatterometer, winds.

\section{INTRODUCTION}

$\mathbf{E}$ RGS are large expanses of sand in the desert. Aeolian processes continue to reshape the erg bedforms through erosion, transportation, and deposition of sand particles. This results in a variety of bedforms. The surface features of these bedforms depend upon the variability of wind speed and wind direction [1]-[4]. Erg surfaces exhibit two scales of roughness features: large-scale sand dunes and small-scale ripples. Ripples have a wavelength of $1-25 \mathrm{~cm}$ and an amplitude of $0.2-5 \mathrm{~cm}$ that result from the short-term wind variations. Sand dunes are 3-600 $\mathrm{m}$ in wavelength and 0.1-100 m amplitude and depend upon the long-term (annual and multiannual) characteristics of winds [5]. In general, both small- and large-scale features of sand bedform consist of facets with slope less than or equal to the angle of repose of sand $\left(30^{\circ}-35^{\circ}\right)$. A facet with slope equal to the angle of repose of sand is called a slipside. Slip-sides are formed in the directions of the prevailing winds. These directions correspond to the modes of the annual directional distribution of the prevailing winds. Large-scale dune structures have one (transverse dunes), two (longitudinal dunes), or more (star dunes) slip-sides where each slip-side

Manuscript received May 17, 2006; revised November 7, 2006.

The authors are with the Center for Earth Remote Sensing, Brigham Young University, Provo, UT 84602 USA (e-mail: hs92@ee.byu.edu; long@ ee.byu.edu).

Digital Object Identifier 10.1109/TGRS.2007.892584

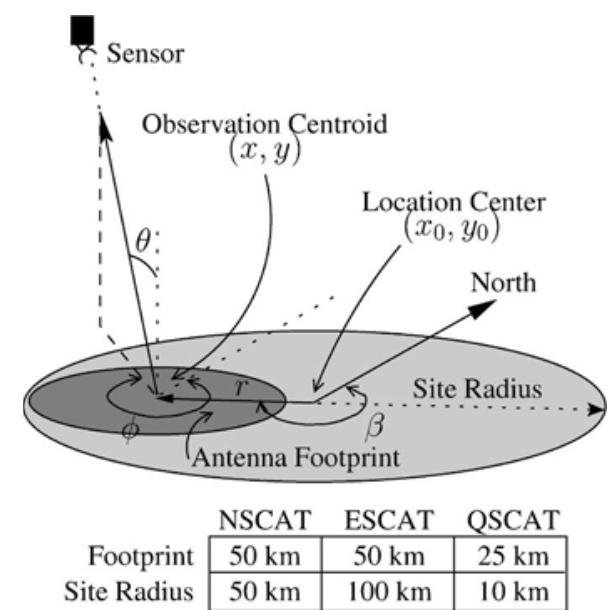

Fig. 1. $\sigma^{\circ}$ observation geometry parameters for a single resolution element within a circular area centered at $\left(x_{0}, y_{0}\right)$. The footprint size and site radius are listed for each sensor.

corresponds to a dominant wind direction. Small-scale ripples are skewed in the direction of wind where the lee-side slopes are steeper than windward-side slopes. These ripples are periodic in the direction of wind.

The NASA Scatterometer (NSCAT) [6], the European Remote Sensing Satellite Scatterometer (ESCAT) [7], and the SeaWinds Scatterometer (QSCAT) aboard QuikScat [8] were initially designed to retrieve near-surface wind fields over the ocean surface. They also observe land surfaces. Over erg surfaces, the backscattering $\left(\sigma^{\circ}\right)$ measured by these instruments depends upon the wind-dependent sand surface geometrical characteristics and instrument observation geometry parameters. The observation geometry parameters are incidence angle $(\theta)$, azimuth angle $(\phi)$ (sensor-target view angles), and antenna footprint centroid $(x, y)$ (target location) [see Fig. 1]. The erg surface geometry varies with time and has a complex dependence on the variability of prevailing winds. Thus, $\sigma^{\circ}$ measurements are also a function of observation time. $\sigma^{\circ} \phi$-modulation reflects the orientation of dominant facets of the large-scale features.

Previous studies show that NSCAT and ESCAT $\sigma^{\circ}$ observations exhibit coupled $\theta$ - and $\phi$-modulation which depends upon the sand surface geometrical characteristics [5], [9]. Over the sand surface, $\theta$ - and $\phi$-modulation have been separately modeled with quadratic and second-order harmonic equations, respectively. Maxima in $\theta$ - and $\phi$-modulation have been related to the slopes and orientations of dominant facets in 
the erg bedform, respectively [10]. Other studies show that the sand surface geometry also effects the $\phi$-modulation of spaceborne radiometric temperature measurements [11] and that $\sigma^{\circ} \phi$-modulation is related to the surface gradient in Greenland [12].

In previous studies, $\sigma^{\circ}$ in the proximity of the point of interest $\left(x_{0}, y_{0}\right)$ is assumed to be homogeneous. This assumption does not apply in the areas of high spatial inhomogeneity such as Greenland [12] and Saharan ergs [13]. In such areas, $\sigma^{\circ}$ varies with the displacement of observation centroid from the study center point $\left(x_{0}, y_{0}\right)$, herein termed as "centroid displacement variability." We define $(r, \beta)$ as the observation displacement from $\left(x_{0}, y_{0}\right)$ where $r$ is the distance (in kilometers) of the observation centroid and $\beta$ is the bearing angle from $\left(x_{0}, y_{0}\right)$ as shown in Fig. 1 .

The European Centre for Medium-Range Weather Forecasts (ECMWF) provides global wind fields generated by a numerical weather prediction model using meteorological data as input. The input wind data are acquired from various sources such as meteorological stations and spaceborne sensors. The predicted winds are spatial and temporal interpolations via a complex global weather model [14].

In this paper, we use vertical polarization (V-pol) $\sigma^{\circ}$ measurements from NSCAT, ESCAT, and QSCAT to study the $\sigma^{\circ}$ response to variations in observation geometry parameters. The temporal variability of both $\sigma^{\circ}$ spatial inhomogeneity and $\phi$-modulation are used to investigate short- and long-term behavior of sand bedforms. We use a second-order harmonic model for $\sigma^{\circ} \phi$-modulation and a variable coefficient secondorder harmonic model for centroid displacement variability. Due to the complicated dependence of $\sigma^{\circ}$ on $\theta$ over the ergs, we compute the model parameters piecewise for different $\theta$ bins. $\sigma^{\circ}$ centroid displacement variability is related to the spatial inhomogeneity of the underlying surface. ECMWF predicted wind vector data are related to the $\sigma^{\circ}$ temporal behavior of the $\phi$-modulation. We investigate the inherent difficulties in modeling $\sigma^{\circ}$ wind response over sand surface.

In order to analyze the $\sigma^{\circ}$ variability with observation geometry over different composite surfaces, 19 sites with known surface types in the Saharo-Arabian Desert are selected. Fig. 2(a) shows the spatial map of the selected points and the corresponding ECMWF annual wind direction distributions at the selected locations during Julian Day (JD) 256, 1996, and JD 256, 1997. Table I describes the surface characteristics of the selected locations reported in [15] and the wind modes estimated from ECMWF predicted winds. The winds with high and low directional variability about the mode are categorized as wide ("WD") and narrow ("NR") mode winds, respectively. This categorization is done arbitrarily by inspection of the annual wind direction histograms. Slip-side orientations computed from NSCAT and ESCAT $\sigma^{\circ} \phi$-modulation at $\theta=33^{\circ}$ are also listed in Table I and discussed in later sections. NSCAT $\sigma^{\circ}$ measurements for which the centroid of the measurement footprint is within $50 \mathrm{~km}$ of each of the selected locations are used. In case of ESCAT and QSCAT, 100- and 10-km radii are used, respectively. $\sigma^{\circ}$ variability induced by the temporal variations in the sand bedform is reduced by using data from a narrow time window. We use $\sigma^{\circ}$ measurements from a 30-day temporal

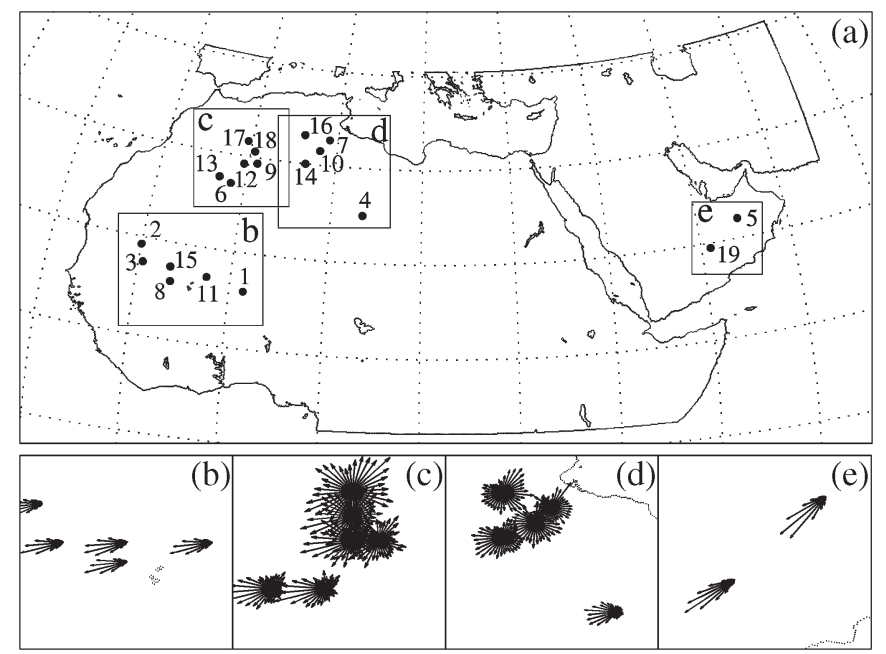

Fig. 2. (a) Map showing the selected points of study and (b)-(e) their annual wind direction distributions (sand roses) during JD 256, 1996, and JD 256, 1997 predicted by ECMWF.

window, effectively assuming that surface characteristics do not change significantly during this time. The effectiveness of this time window diminishes in the areas of highly variable winds. The selected spatial radii for each instrument and temporal window size provide sufficient measurement samples to estimate the model parameters. The model parameters are computed every 15 days with overlapping windows to study seasonal effects.

In Section II, we introduce a comprehensive model of $\sigma^{\circ}$ modulation as a function of observation geometry. The $\sigma^{\circ}$ variability due to view angle and measurement centroid displacement are addressed. In Section III, model inversion results are presented to understand and relate the model parameters to the surface characteristics. The dependence of model parameters on $\theta$ over different sand surfaces is presented. The spatial and temporal behaviors of model parameters in comparison to ECMWF winds are also given. The scope and limitations of this method are also described. Section IV provides a summary and conclusion.

\section{OBSERVATION GeOMETRy-InduCED $\sigma^{\circ}$ MOdulation}

In this section, the variability induced in $\sigma^{\circ}$ measurements due to varying parameters of the observation geometry illustrated in Fig. 1 is presented. Over sand surfaces, the $\sigma^{\circ}$ measurements (in decibels) as a function of observation geometry are modeled as

$$
\sigma^{\circ}(\theta, \phi, r, \beta)=A_{\theta}+q_{\theta}(\phi)+h_{\theta}(r, \beta)
$$

where $\sigma^{\circ}(\theta, \phi, r, \beta)$ is the scatterometer measurement with view angles $(\theta, \phi)$ and the centroid of its measurement footprint at $(r, \beta)$ from $\left(x_{0}, y_{0}\right)$. The dependence of the model terms on incidence angle is represented by the subscript $\theta . A_{\theta}$ is the mean response of the surface which depends upon $\theta . q_{\theta}(\phi)$ and $h_{\theta}(r, \beta)$ are the observation azimuth angle modulation and observation centroid displacement variability, respectively, as a function of $\theta$. In this model, the time dependence is neglected by selecting a narrow time window. 
TABLE I

Table Listing the Surface Types and ECMWF Wind Modes Over the Selected Study Sites. Narrow and Wide Wind MOdes ARE SYMBOLIZED B “NR” AND “WD,” RESPECTIVELY. THE ORIENTATIONS OF SLip-Sides Estimated From Two Scatterometers Are Also Given

\begin{tabular}{|c|c|c|c|c|c|}
\hline \multirow{2}{*}{ \# } & \multirow{2}{*}{ Location } & \multirow{2}{*}{ Surface Dune Types } & \multirow{2}{*}{ ECMWF Winds } & \multicolumn{2}{|c|}{$q_{\theta}(\phi)$ Peaks at $\theta=33^{\circ}$} \\
\hline & & & & NSCAT & ESCAT \\
\hline$\overline{1}$ & $16.00^{\circ} \mathrm{N}, 1.50^{\circ} \mathrm{E}$ & Barchans & $255^{\circ} \mathrm{NR}$ & $289^{\circ}$ & $250^{\circ}$ \\
\hline 2 & $19.25^{\circ} \mathrm{N}, 10.00^{\circ} \mathrm{W}$ & Barchanoid Ridge & $255^{\circ} \mathrm{NR}$ & $271^{\circ}$ & $278^{\circ}$ \\
\hline 3 & $17.50^{\circ} \mathrm{N}, 9.50^{\circ} \mathrm{W}$ & Barchanoid Ridge & $255^{\circ} \mathrm{NR}$ & $258^{\circ}$ & $164^{\circ}$ \\
\hline 4 & $25.00^{\circ} \mathrm{N}, 13.50^{\circ} \mathrm{E}$ & Barchanoid Ridge & $274^{\circ} \mathrm{WD}$ & $104^{\circ}$ & $113^{\circ}$ \\
\hline 5 & $22.00^{\circ} \mathrm{N}, 54.50^{\circ} \mathrm{E}$ & Barchanoid Ridge & $228^{\circ} \mathrm{NR}$ & $297^{\circ}$ & $291^{\circ}$ \\
\hline 6 & $27.00^{\circ} \mathrm{N}, 1.60^{\circ} \mathrm{W}$ & Crescent Dunes Superimposed & $255^{\circ} \mathrm{WD}, 45^{\circ} \mathrm{WD}$ & $213^{\circ} \& 44^{\circ}$ & $240^{\circ} \& 71^{\circ}$ \\
\hline 7 & $32.70^{\circ} \mathrm{N}, 9.00^{\circ} \mathrm{E}$ & Fish-scale Pattern & Multimodal & $290^{\circ} \& 115^{\circ}$ & $254^{\circ} \& 107^{\circ}$ \\
\hline 8 & $16.00^{\circ} \mathrm{N}, 6.25^{\circ} \mathrm{W}$ & Linear Dunes & $255^{\circ} \mathrm{NR}$ & $266^{\circ}$ & $285^{\circ}$ \\
\hline 9 & $29.40^{\circ} \mathrm{N}, 1.00^{\circ} \mathrm{E}$ & Linear Dunes & $265^{\circ} \mathrm{WD}, 36^{\circ} \mathrm{WD}$ & $228^{\circ} \& 37^{\circ}$ & $216^{\circ} \& 56^{\circ}$ \\
\hline 10 & $31.50^{\circ} \mathrm{N}, 8.00^{\circ} \mathrm{E}$ & Linear Dunes & Multimodal & $192^{\circ} \& 25^{\circ}$ & $286^{\circ} \& 108^{\circ}$ \\
\hline 11 & $17.00^{\circ} \mathrm{N}, 2.50^{\circ} \mathrm{W}$ & Compound Linear Dunes & $254^{\circ} \mathrm{NR}$ & $262^{\circ}$ & $274^{\circ}$ \\
\hline 12 & $29.20^{\circ} \mathrm{N}, 0.50^{\circ} \mathrm{W}$ & Compound Linear Dunes & $255^{\circ} \mathrm{WD}, 35^{\circ} \mathrm{WD}$ & $227^{\circ} \& 340^{\circ}$ & $201^{\circ} \& 48^{\circ}$ \\
\hline 13 & $27.50^{\circ} \mathrm{N}, 3.00^{\circ} \mathrm{W}$ & Star Dunes Superimposed & $255^{\circ} \mathrm{WD}, 35^{\circ} \mathrm{WD}$ & $268^{\circ} \& 71^{\circ}$ & $263^{\circ} \& 77^{\circ}$ \\
\hline 14 & $30.00^{\circ} \mathrm{N}, 6.50^{\circ} \mathrm{E}$ & Chain Star Dunes & $235^{\circ} \mathrm{WD}, 35^{\circ} \mathrm{WD}$ & $351^{\circ} \& 173^{\circ}$ & $266^{\circ} \& 77^{\circ}$ \\
\hline 15 & $17.50^{\circ} \mathrm{N}, 6.50^{\circ} \mathrm{W}$ & Sand Sheets Streaks & $255^{\circ} \mathrm{NR}$ & $259^{\circ}$ & $265^{\circ}$ \\
\hline 16 & $33.00^{\circ} \mathrm{N}, 6.00^{\circ} \mathrm{E}$ & Sand Sheets Streaks & $35^{\circ} \mathrm{WD}$ & $118^{\circ}$ & $89^{\circ}$ \\
\hline 17 & $31.60^{\circ} \mathrm{N}, 0.50^{\circ} \mathrm{W}$ & Compound Sand Sheets Streaks & $45^{\circ} \mathrm{WD} 244^{\circ} \mathrm{WD}$ & $28^{\circ} \& 222^{\circ}$ & $71^{\circ} \& 270^{\circ}$ \\
\hline 18 & $30.60^{\circ} \mathrm{N}, 0.50^{\circ} \mathrm{E}$ & Undifferentiated Complex Dunes & $255^{\circ} \mathrm{WD}, 24^{\circ} \mathrm{WD}$ & $189^{\circ} \& 2^{\circ}$ & $250^{\circ} \& 54^{\circ}$ \\
\hline 19 & $19.50^{\circ} \mathrm{N}, 51.00^{\circ} \mathrm{E}$ & Undifferentiated Complex Dunes & $235^{\circ} \mathrm{NR}$ & $354^{\circ}$ & $300^{\circ}$ \\
\hline
\end{tabular}

In previous studies, $\sigma^{\circ} \theta$ dependence has been modeled with linear and quadratic dependence [9]. The quadratic model adequately models $\theta$ dependence over erg sand sheets, which are approximately a single facet. The response over tilted sand sheets is similar, but shifted in $\theta$ depending upon the local incidence angle $\left(\theta^{\prime}\right)$, which is defined as the angle between view direction and facet's local normal. $\theta^{\prime}$ is a function of facet tilt and sensor view direction. The tilted facet of a dune has maximum backscatter when $\theta^{\prime}$ is minimum [10].

Due fields are composed of multiple tilted facets and result in a complicated $\sigma^{\circ} \theta$ dependence as revealed in Fig. 3. In such areas, the $\sigma^{\circ}$ response is the sum of responses from individual facets at various $\theta^{\prime}$, weighted by the facet's area projected in the direction of the sensor [10]. Note that the $\sigma^{\circ}$ variation with $\theta$ is different for different dunes. Thus, we compute model parameters separately for different $\theta$ bins. The model in (1) states that for a given $\theta$, the observed $\sigma^{\circ}$ is given by the sum of the mean surface response, a $\phi$-modulation, and the variability due to measurement centroid. In the next two sections, we discuss the observation $\phi$-modulation and centroid displacement variability.

\section{A. $\sigma^{\circ}$ Azimuth Angle Modulation}

NSCAT and ESCAT provide $\sigma^{\circ}$ measurements at multiple $\theta$ and $\phi$ angles. $\sigma^{\circ}$ from a flat sand surface is maximum at near-nadir, whereas a tilted surface with slope $\theta_{\mathrm{s}}$ and orientation $\phi_{\mathrm{s}}$ has maximum backscatter when $\theta=\theta_{\mathrm{s}}$ and $\phi=\phi_{\mathrm{s}}$. Thus, the presence of large-scale slip-sides results in a strong $\sigma^{\circ} \phi$-modulation return at $\theta=30^{\circ}-35^{\circ}$. The azimuth angle at which the maximum of the $\phi$-modulation occurs is equal to the orientation of the slip-side, which is related to the dominant wind direction [10].

NSCAT and ESCAT measure V-pol $\sigma^{\circ}$ at ten and six azimuth angles, respectively. QSCAT V-pol $\sigma^{\circ}$ measurements have rela-

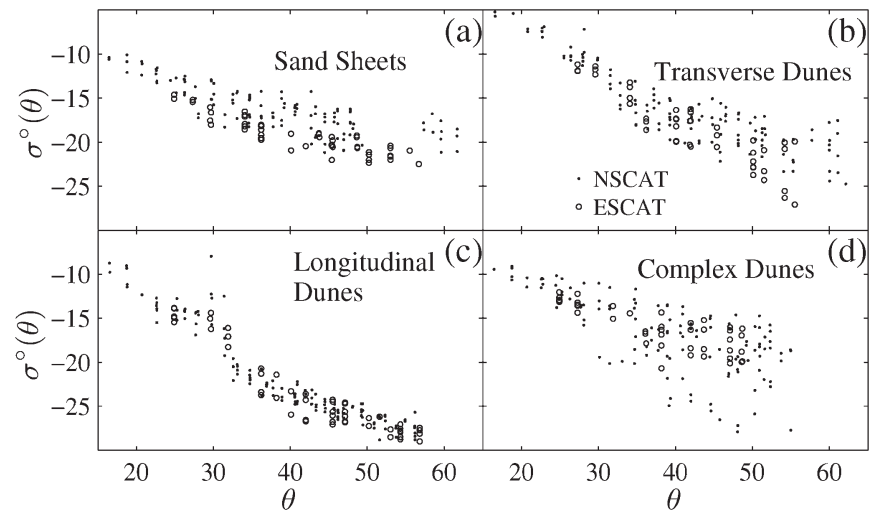

Fig. 3. NSCAT and ESCAT $\sigma^{\circ}(\theta)$ at $\phi=127^{\circ}$ during JD 1-31, 1997, for (a) sand sheets, (b) transverse dunes, (c) longitudinal dunes, and (d) complex dunes corresponding to locations 5, 10, 16, and 18 .

tively higher azimuth sampling, but only at one incidence angle $\left(54^{\circ}\right)$. Previous studies use a second-order harmonic model to represent the $\sigma^{\circ} \phi$-modulation [9] without accounting for incidence angle dependence, i.e.,

$$
q(\phi)=M_{1} \cos \left(\phi-\phi_{1}\right)+M_{2} \cos \left(2 \phi-\phi_{2}\right)
$$

where $M_{i}$ and $\phi_{i}$ are the magnitude and the phase of the $i$ thorder harmonic. Over dune surfaces, $M_{i}$ and $\phi_{i}$ are a function of $\theta$. Due to a complicated dependence on the incidence angle, we use the second-order harmonic model separately for each $\theta$ given by

$$
q_{\theta}(\phi)=M_{\theta 1} \cos \left(\phi-\phi_{\theta 1}\right)+M_{\theta 2} \cos \left[2\left(\phi-\phi_{\theta 2}\right)\right]
$$

where $M_{\theta i}$ and $\phi_{\theta i}$ are the magnitude and phase, respectively, of the $i$ th harmonic computed for incidence angle $\theta$. The quality of this model fit depends upon the $\theta$ and $\phi$ sampling density of $\sigma^{\circ}$ measurements. Fig. 4(a) and (c) shows the $\sigma^{\circ}$ measurement 

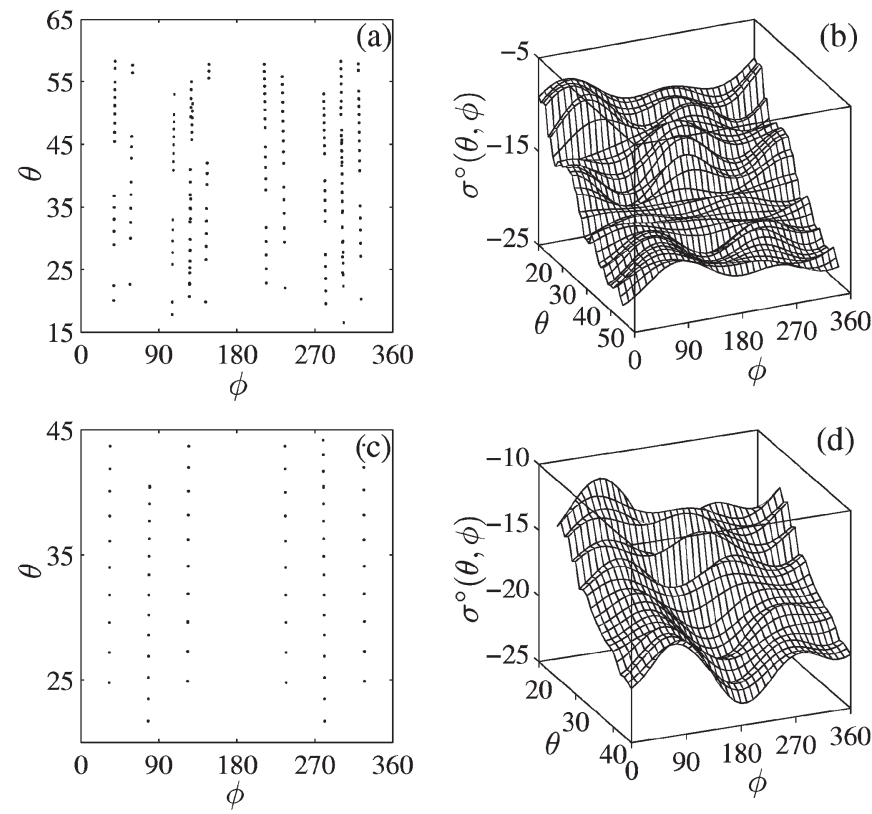

Fig. 4. (a) and (c) Plots of $\theta$ and $\phi$ for each measurement during JD 1-30, 1997, at location 18 from NSCAT and ESCAT, respectively. (b) and (d) $\sigma^{\circ}(\theta, \phi)$ model surface fits as a function of $\theta$ and $\phi$ from NSCAT and ESCAT, respectively. The rms errors of model fits for NSCAT and ESCAT are 3.63 and $2.97 \mathrm{~dB}$, respectively.

$\theta$ and $\phi$ sampling of NSCAT and ESCAT, respectively, over location 18 with complex dunes. In order to estimate the model parameters at a given $\theta, \sigma^{\circ}$ measurements with incidence angles within $\theta \pm 3.5^{\circ}$ and a $7^{\circ}$ wide Gaussian weighting kernel centered at the given $\theta$ are used to perform a weighted least squares fit.

Fig. 4(b) and (d) shows the $\sigma^{\circ}(\theta, \phi)=A_{\theta}+q_{\theta}(\phi)$ model fits to NSCAT and ESCAT data, respectively. Fig. 6(b) and (d) shows, for the respective data, slices through these surface fits and the corresponding raw measurements. Other plots in Fig. 6 are explained in Section III. Table II lists the rms errors of the model applied to NSCAT and ESCAT data over each of the study locations. RMS errors of other models are discussed in the following sections. The incidence-angle-dependent model $q_{\theta}(\phi)$ has lower rms error than $q(\phi)$ (where $\theta$ is not considered) and its surface fit provides a comprehensive view of the $\sigma^{\circ}$ dependence on the view angles. The reduction in rms error is similar at all locations. The locations with high rms error have high surface inhomogeneity not accounted for in these two models. Although the $\phi$-modulation is adequately modeled, there exists data variance due to the centroid displacement variability discussed in the next section.

Fig. 4 reveals that the model parameters change significantly with incidence angle. The difference in the $\phi$-modulation at lower and higher incidence angles depends upon the sand surface geometry. The wind-induced changes in large- and small-scale surface geometry add, remove, and/or shift peaks in the $\phi$-modulation. Generally, short-term changes in the winds are reflected by the changes in the small-scale surface ripples, which are periodic in the wind direction. This alignment of surface ripples to the wind becomes complicated over tilted facets of large-scale dunes. Orographic wind flow over the tilted dune facets may deviate from the average prevailing wind.
Thus, the orientation of small-scale ripples on a facet may not align exactly with the average wind direction. Since the simple model presented in this paper relies on the $\sigma^{\circ}$ dependence on surface geometry to infer average winds, its accuracy reduces over the areas with complex sand bedforms.

\section{B. $\sigma^{\circ}$ Centroid Displacement Variability}

$\sigma^{\circ}$ varies with the centroid displacement due to the surface spatial variability. This variability reflects the inhomogeneity of the underlying surface, which in the case of the ergs is caused by varying dune shapes, dune density, sand depth, and largescale slope. Compound dune fields and mixed dune fields have varying dune shapes and spatial densities. The varying sand depth also introduces variations in the $\theta$ dependence of the displacement variability. The varying large-scale slope from the middle to the fringes of the erg basins appear to play a significant role in the centroid displacement variability. At the fringes of the ergs, the surface topography transitions from a sandy to a rocky surface and results in maximum $\sigma^{\circ}$ spatial inhomogeneity.

Analysis of $\sigma^{\circ}$ observations over various locations suggests that the $\sigma^{\circ}$ centroid displacement variability $[h(r, \beta)]$ is best modeled by a variable coefficient second-order harmonic equation given by

$$
h(r, \beta)=H_{1} r \cos \left(\beta-\beta_{1}\right)+H_{2} r^{2} \cos \left[2\left(\beta-\beta_{2}\right)\right]
$$

where the first term on the right side is a plane with the steepest ascent of $H_{1}(\mathrm{~dB} / \mathrm{km})$ in the direction $\beta_{1}$ and the second term is a hyperbolic paraboloid with the highest curvature $H_{2}\left(\mathrm{~dB} / \mathrm{km}^{2}\right)$ in the direction $\beta_{2} . H_{1}$ and $H_{2}$ represent the magnitudes of a plane and a hyperbolic paraboloid, linearly combined and added to the mean response.

Fig. 5(a) shows the $\sigma^{\circ}(r, \beta)=A+h(r, \beta)$ model fit ( $A$ is the mean $\sigma^{\circ}$ with no $\theta$ dependence) to NSCAT $\sigma^{\circ}$ measurements with an rms error of $4.21 \mathrm{~dB}$. Two slices through the fit at $r=15 \mathrm{~km}$ and $r=30 \mathrm{~km}$ are shown in Fig. 5(b). Their comparison to the $\sigma^{\circ}$ measurements within $3 \mathrm{~km}$ of these radii show that $h(r, \beta)$ alone does not represent the behavior of all data measurements. By taking into account the strong dependence of $\sigma^{\circ}$ on $\theta$, the model fit is improved by computing the model parameters separately for different incidence angles using

$$
h_{\theta}(r, \beta)=H_{\theta 1} r \cos \left(\beta-\beta_{\theta 1}\right)+H_{\theta 2} r^{2} \cos \left[2\left(\beta-\beta_{\theta 2}\right)\right]
$$

where the subscript $\theta$ of model parameters represents their $\theta$ dependence. Fig. 5(c) and (e) gives the model fit of $\sigma^{\circ}(\theta, r, \beta)=$ $A_{\theta}+h_{\theta}(r, \beta)$ for $\theta$ ranges $25^{\circ}-35^{\circ}$ and $40^{\circ}-50^{\circ}$. Comparison reveals the strong incidence angle dependence of the $\sigma^{\circ}$ spatial inhomogeneity. Table II, fifth and sixth columns, presents the rms errors of $h(r, \beta)$ and $h_{\theta}(r, \beta)$ model fits to the NSCAT $\sigma^{\circ}$ measurements over the selected locations. The incidentangle-dependent model significantly reduces the rms error over complex bedforms. The rms errors of fits to ESCAT data show similar behavior. These results confirm that $\theta$-dependent $\sigma^{\circ}$ spatial inhomogeneity is significant over the complex erg bedforms. 
TABLE II

RMS ERrors for Different Model Versions Applied to NSCAT ANd ESCAT DATA AT 19 LocAtions During JD 1-30, 1997

\begin{tabular}{|c|c|c|c|c|c||c|c|c|c|c|}
\hline \multirow{2}{*}{ Site \# } & \multicolumn{9}{|c||}{ NSCAT } & \multicolumn{5}{c|}{ ESCAT } \\
\cline { 2 - 11 } & $q(\phi)$ & $q_{\theta}(\phi)$ & $h(r, \beta)$ & $h_{\theta}(r, \beta)$ & $q_{\theta}(\phi)+h_{\theta}(r, \beta)$ & $q(\phi)$ & $q_{\theta}(\phi)$ & $h(r, \beta)$ & $h_{\theta}(r, \beta)$ & $q_{\theta}(\phi)+h_{\theta}(r, \beta)$ \\
\hline \hline 1 & 2.25 & 0.97 & 2.19 & 0.69 & 0.68 & 1.41 & 0.74 & 1.39 & 0.54 & 0.55 \\
\hline 2 & 4.48 & 2.16 & 4.46 & 1.74 & 1.48 & 4.60 & 1.89 & 4.70 & 2.26 & 1.47 \\
\hline 3 & 3.69 & 1.09 & 3.70 & 1.37 & 0.95 & 3.42 & 1.11 & 3.49 & 1.48 & 1.00 \\
\hline 4 & 5.20 & 2.21 & 5.20 & 2.52 & 1.54 & 4.26 & 2.52 & 4.21 & 2.49 & 1.86 \\
\hline 5 & 4.80 & 1.64 & 5.21 & 1.82 & 1.47 & 5.32 & 1.80 & 5.33 & 1.97 & 1.61 \\
\hline 6 & 3.55 & 2.27 & 3.03 & 1.40 & 1.33 & 2.72 & 1.59 & 2.20 & 1.03 & 0.90 \\
\hline 7 & 6.07 & 1.95 & 6.25 & 1.94 & 1.65 & 3.95 & 2.37 & 3.77 & 1.70 & 1.47 \\
\hline 8 & 2.61 & 1.49 & 2.33 & 0.64 & 0.63 & 1.88 & 1.47 & 1.37 & 0.48 & 0.48 \\
\hline 9 & 2.93 & 1.92 & 2.57 & 1.17 & 1.14 & 2.86 & 2.42 & 2.07 & 1.01 & 0.90 \\
\hline 10 & 5.69 & 1.25 & 5.80 & 1.44 & 1.17 & 4.23 & 1.22 & 4.44 & 1.30 & 1.04 \\
\hline 11 & 3.38 & 1.04 & 3.37 & 0.77 & 0.74 & 2.28 & 1.34 & 2.02 & 0.68 & 0.64 \\
\hline 12 & 5.54 & 3.99 & 4.61 & 1.98 & 1.81 & 3.56 & 2.42 & 3.15 & 1.24 & 1.07 \\
\hline 13 & 3.59 & 1.79 & 3.36 & 1.08 & 1.08 & 2.97 & 1.84 & 2.57 & 0.93 & 0.86 \\
\hline 14 & 3.17 & 1.08 & 3.18 & 0.80 & 0.77 & 3.60 & 1.95 & 3.49 & 1.04 & 0.98 \\
\hline 15 & 3.45 & 1.87 & 3.40 & 1.77 & 1.24 & 3.37 & 1.79 & 3.62 & 1.82 & 1.32 \\
\hline 16 & 3.18 & 2.16 & 2.84 & 1.61 & 1.39 & 2.86 & 2.25 & 2.27 & 0.85 & 0.79 \\
\hline 17 & 2.48 & 0.87 & 2.44 & 0.65 & 0.63 & 1.41 & 0.86 & 1.23 & 0.58 & 0.54 \\
\hline 18 & 5.09 & 3.63 & 4.21 & 1.75 & 1.58 & 4.01 & 2.97 & 3.65 & 1.63 & 1.41 \\
\hline 19 & 4.74 & 1.34 & 5.20 & 1.86 & 1.27 & 3.95 & 1.64 & 3.85 & 1.38 & 1.01 \\
\hline
\end{tabular}

(a)
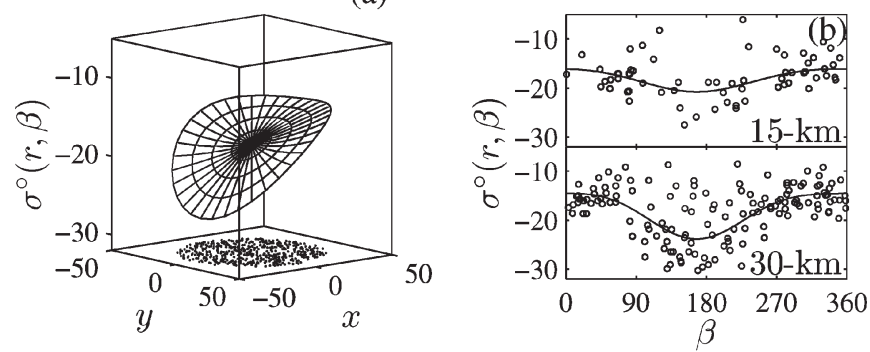

(c)
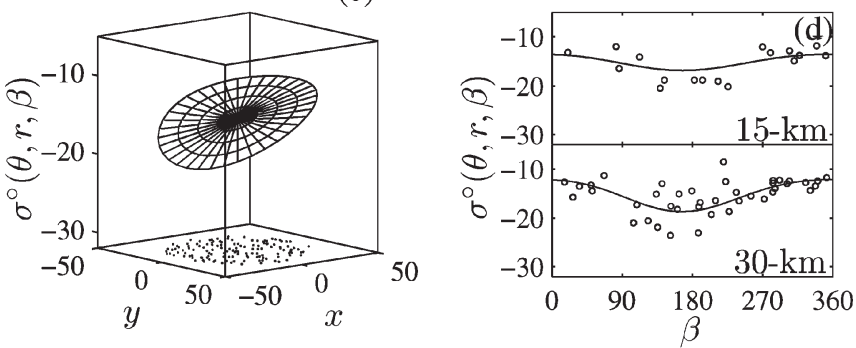

(e)
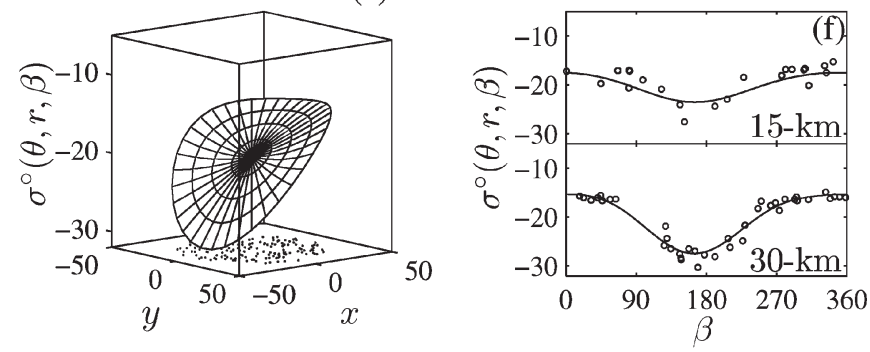

Fig. 5. (a) $\sigma^{\circ}(r, \beta)$ model fit to NSCAT $\sigma^{\circ}$ measurements for incidence angles $17^{\circ}-58^{\circ}$ during JD $1-30,1997$, at location 18 . The centroid locations of measurements within $50 \mathrm{~km}$ of location 18 are shown at the bottom of the box to indicate the density of spatial samples used in the model fit. (b) Two slices through the spatial model fit at $r=15 \mathrm{~km}$ and $r=30 \mathrm{~km}$ from the center. Raw $\sigma^{\circ}$ measurements within $3 \mathrm{~km}$ of each radius are also shown to analyze the quality of the model fit. (c)-(f) Similar plots for measurements with incidence angle ranges $25^{\circ}-35^{\circ}$ and $40^{\circ}-50^{\circ}$. The rms error of the model fits in (a), (c), and (e) are $4.21,2.45$, and $1.87 \mathrm{~dB}$, respectively.

\section{III. $\sigma^{\circ}$ Full OBservation Model Results}

In the following analysis, the full observation geometry model in (1) is used with the incidence-angle-dependent azimuth angle modulation and centroid displacement variability models given in the last two sections. This model is applied to the NSCAT and ESCAT data used in Fig. 4. The model fit results are shown in Fig. 6. The full model shows a significant improvement in the fit by removing the $\sigma^{\circ}$ spatial inhomogeneity. This improvement is consistent in all scatterometers, but is most obvious in the NSCAT data at $\theta=37^{\circ}$. The surface fits achieved with the full observation model reduce the rms error for NSCAT and ESCAT to 1.58 and $1.41 \mathrm{~dB}$, from 3.63 and $2.97 \mathrm{~dB}$ when spatial dependence is ignored [see Table II]. The corresponding QSCAT data, only available at $\theta=54^{\circ}$, result in rms errors of 0.6 and $1.57 \mathrm{~dB}$, with and without considering spatial inhomogeneity.

Table II lists the full observation model rms errors over all selected locations for NSCAT and ESCAT. The full observation model reduces the rms fit error by more than $2 \mathrm{~dB}$ at locations 12 and 18 which are highly inhomogeneous bedforms. In relatively homogenous locations such as 1, 3, 5, 10, and 17, rms error reduction is less than $0.5 \mathrm{~dB}$, confirming the expected spatial homogeneity.

\section{A. Incidence Angle Dependence of Model Parameters}

The incidence angle dependence of the model results in model parameters that are a function of incidence angle. The $\theta$ dependence of model parameters depends upon the nature of the surface geometry. Fig. 7 shows the plots of model parameters as a function of incidence angle for sand sheet, transverse dunes, longitudinal dunes, and complex dunes.

Sand sheets are generally flat erg surfaces with a general slope and small-scale ripples. This results in a less complicated incidence angle dependence of $\sigma^{\circ}$ compared to dunes. In such 


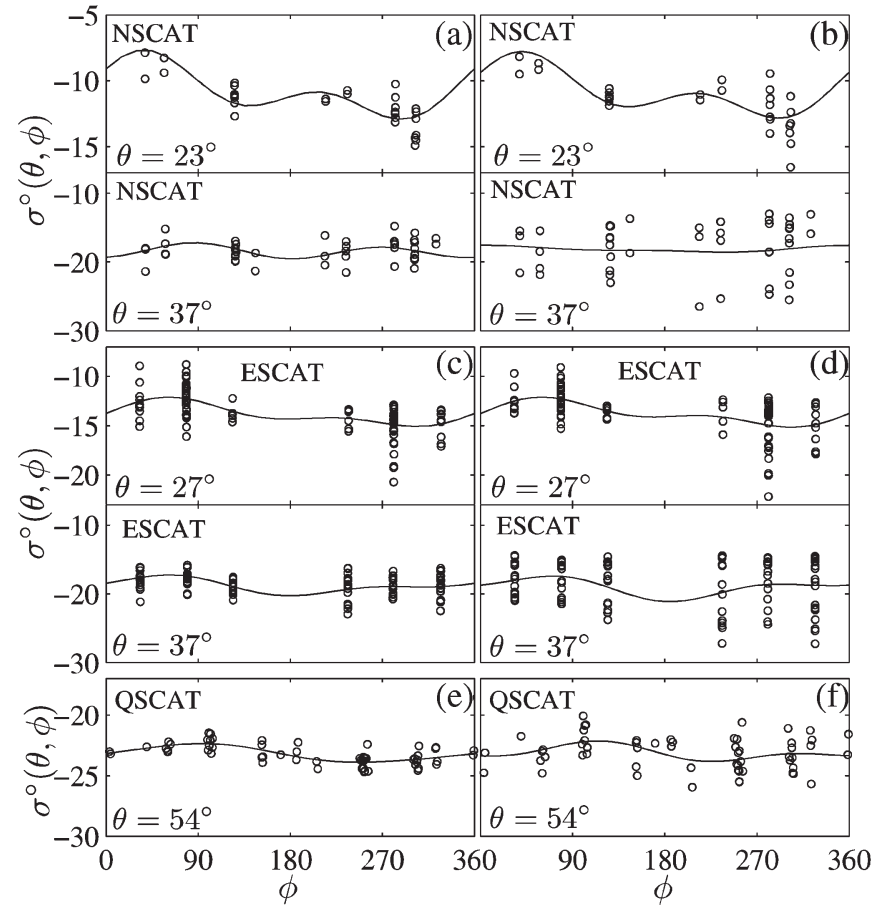

Fig. 6. Model fits with (left) and without (right) considering $\sigma^{\circ}$ spatial inhomogeneity at location 18 . Solid lines are the modeled $\phi$-modulation at selected incidence angles. In the left plots, the raw $\sigma^{\circ}$ measurements within $2^{\circ}$ of selected $\theta$ are shown whereas the right plots show the raw measurements after subtracting the $\sigma^{\circ}$ spatial inhomogeneity term $h_{\theta}(r, \beta)$. (a), (c), and (e) are the results from NSCAT, ESCAT, and QSCAT data, respectively, using the model with the spatial inhomogeneity term. (b), (d), and (f) are the corresponding results from the model without the spatial inhomogeneity term.

areas $\sigma^{\circ}$ mainly depends upon the sand depth (i.e., relative surface and volume scattering at different incidence angles). The first-order $\sigma^{\circ}$ spatial inhomogeneity (plane) has little dependence on the incidence angle and is directed at $310^{\circ}$ with a magnitude $\left(H_{\theta 1}\right)$ of less than $0.1 \mathrm{~dB} / \mathrm{km}$. This spatial inhomogeneity is due to the varying general slope of the sand sheet. The second-order $\sigma^{\circ}$ spatial inhomogeneity (hyperbolic paraboloid) $\left(H_{\theta 2}\right)$ is less than $0.001 \mathrm{~dB} / \mathrm{km}^{2}$ and has a direction almost equal to $\beta_{\theta 1}$. We conjecture that the second-order inhomogeneity is primarily caused by spatially varying small-scale geometry. The $\theta$ dependence of the magnitude of second-order inhomogeneity results from variations in the illumination of the small-scale ripples at different incidence angles. The first- and second-order harmonics of the azimuth angle modulation over sand sheets are caused by the general slope of the surface and small-scale geometry, respectively.

Transverse dunes have a slip-side and a windward-side. The windward-side has a slope of $10^{\circ}-15^{\circ}$. Longitudinal dunes have two facets that are both slip-sides. The $A_{\theta}$ difference between low and high incidence angles over such dune types is higher than sand sheets due to the presence of more than one dominant facet. These facets result in increased $\sigma^{\circ}$ at certain $\theta$ and $\phi$ angles which are related to the slope and orientation of constituting facets. In comparison to sand sheets, transverse and longitudinal dune fields exhibit greater $\theta$ dependence of the magnitudes and phases of the two harmonics. The first-order $\sigma^{\circ}$ spatial inhomogeneity magnitude is about half that of sand

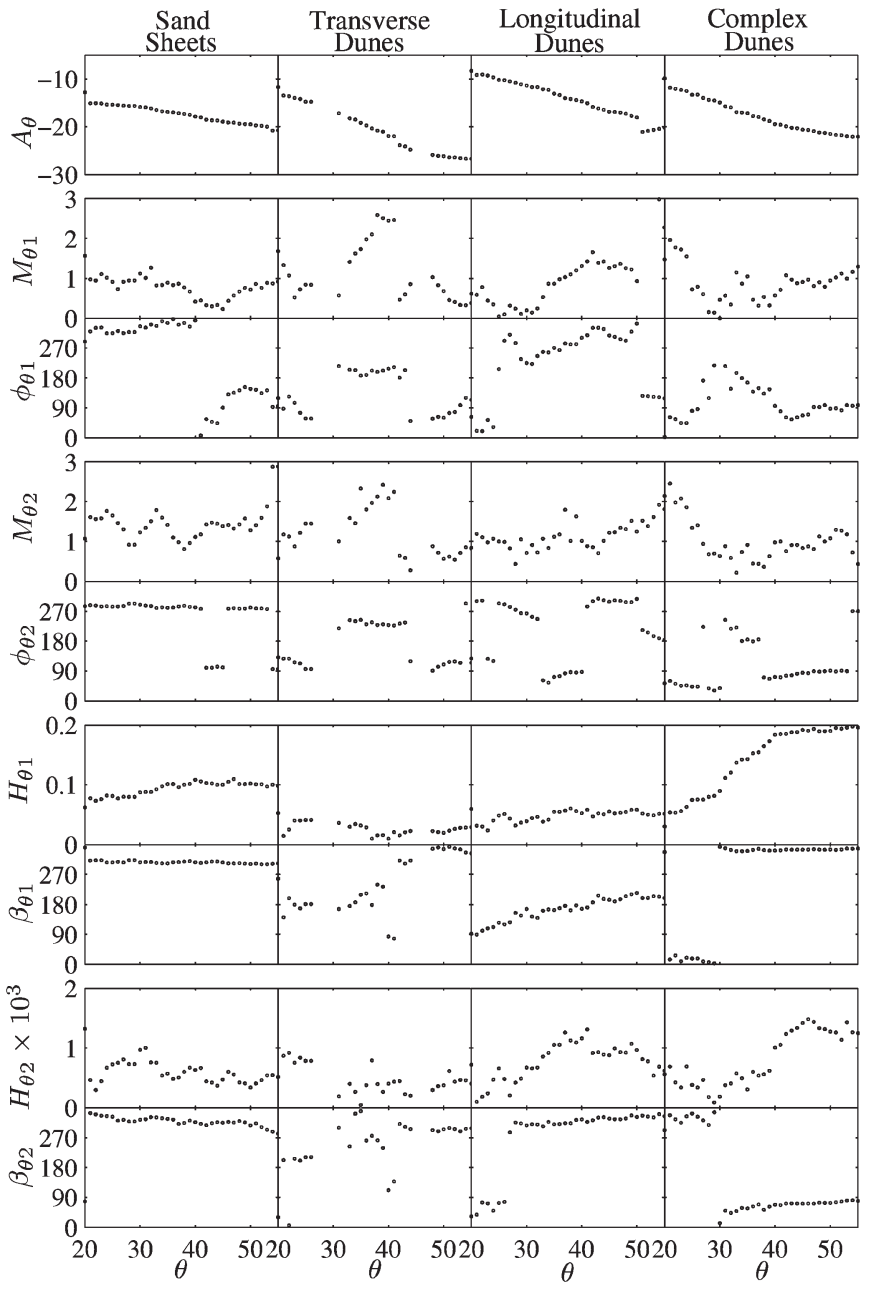

Fig. 7. Plots illustrating the incidence angle dependence of the model parameters for sand sheets (first column), transverse dunes (second column), longitudinal dunes (third column), and complex dunes (fourth column) at locations $16,5,10$, and 18, respectively. The nine rows of the plot array correspond to the model parameters $A_{\theta}, M_{\theta 1}, \phi_{\theta 1}, M_{\theta 2}, \phi_{\theta 2}, H_{\theta 1}, \beta_{\theta 1}$, $H_{\theta 2}$, and $\beta_{\theta 2}$.

sheets whereas the second-order inhomogeneity has magnitude comparable to sand sheets. The directions $\beta_{\theta 1}$ and $\beta_{\theta 2}$ have greater variability with $\theta . \sigma^{\circ}$ spatial inhomogeneity over these dune fields is primarily introduced by spatially varying slopes, orientations, and densities of the dune facets. Spatial variations in the directions of dune axes, merging of dunes, splitting of dunes, and spatial transitions between different types of dune fields also contribute to this inhomogeneity.

Complex dunes have significant spatial variability that results in high $\sigma^{\circ}$ spatial inhomogeneity. The magnitudes of the $\sigma^{\circ}$ spatial inhomogeneity increase with $\theta$ and almost double at the far range incidence angles whereas the directions have little dependence on $\theta$.

$\sigma^{\circ} \theta$-dependent $\phi$-modulation $q_{\theta}(\phi)$ and spatial inhomogeneity $h_{\theta}(r, \beta)$ are computed for locations 5 and 16 and shown in Fig. 8 for $\theta=33^{\circ}$. The sand sheet $q_{\theta}(\phi)$ plot has two peaks at $100^{\circ}$ and $270^{\circ}$ for all incidence angles, indicating a single facet surface. The shape of $h_{\theta}(r, \beta)$ indicates the extent and direction of $\sigma^{\circ}$ spatial inhomogeneity caused by varying slopes and small scale geometry. Other sand surfaces' $q_{\theta}(\phi)$ modulation 

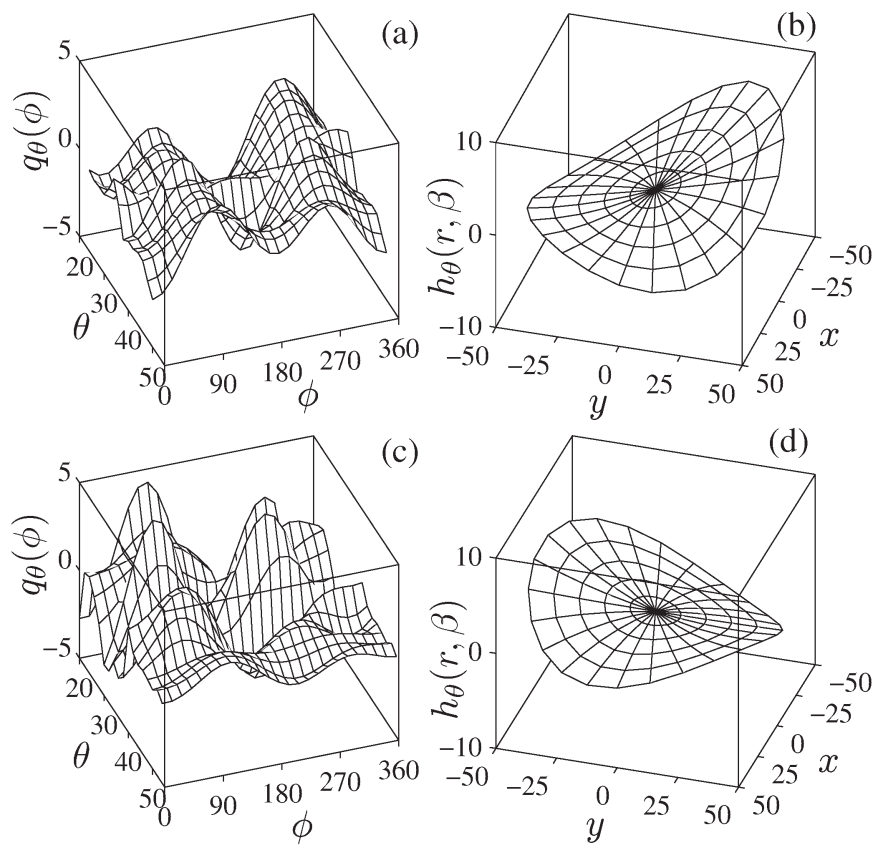

Fig. 8. (a) and (c) Plots of $q_{\theta}(\phi)$ and (b) and (d) $h_{\theta}(r, \beta)$ at $\theta=33^{\circ}$ computed using the full model for (a) and (b) sand sheets and (c) and (d) transverse dunes.
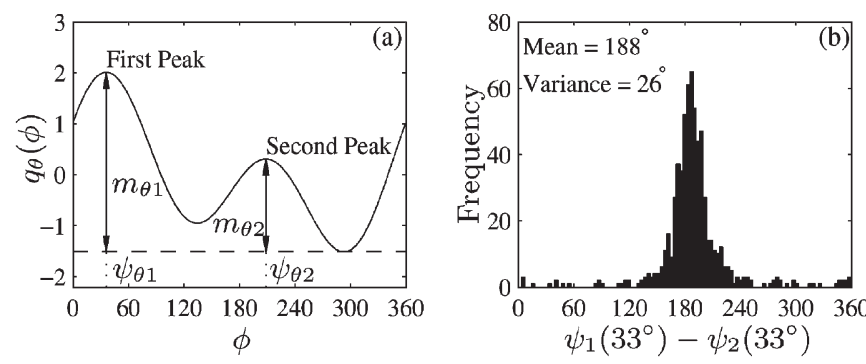

Fig. 9. (a) Slice through $q_{\theta}(\phi)$ surface fit is shown to define azimuth angle of a peak $(\psi)$ and magnitude $(m)$ of $\phi$-modulation at $\psi$. (b) Histogram of angular difference between two peaks over all the ergs showing that, in general, two peaks of $\phi$-modulation are separated by $180^{\circ}$.

significantly changes with the incidence angle. Complex dune $q_{\theta}(\phi)$ has various peaks caused by the dominant facets of these dunes.

The second-order harmonic modulation results in 1-2 maxima depending upon the magnitudes and phases of the two harmonics. We define $\psi$ as the azimuth angle at which the peak occurs and $m$ as the magnitude of modulation at this angle [see Fig. 9(a)]. Over the erg regions, the two peaks at $\psi_{1}$ and $\psi_{2}$ are generally separated by $180^{\circ}$. Fig. 9 (b) shows the histogram of their difference at $\theta=33^{\circ}$ with the mean value of $188^{\circ}$.

The azimuth angle and magnitude of the maxima of $\phi$-modulation depend upon the incidence angle. Fig. 10 shows the plots of $\psi$ and $m$ as a function of $\theta$ for the four sand surface types. There is no dominant peak over the sand sheets. Transverse and longitudinal fields have one and two peaks between $30^{\circ}$ and $35^{\circ}$ incidence angles, respectively. The complex dunes show more complicated behavior. The azimuth angles of peaks of the $\sigma^{\circ} \phi$-modulation at $33^{\circ}$ incidence angle are aligned to the average surface wind direction in the area. This method is used to find slip-side orientations from NSCAT and

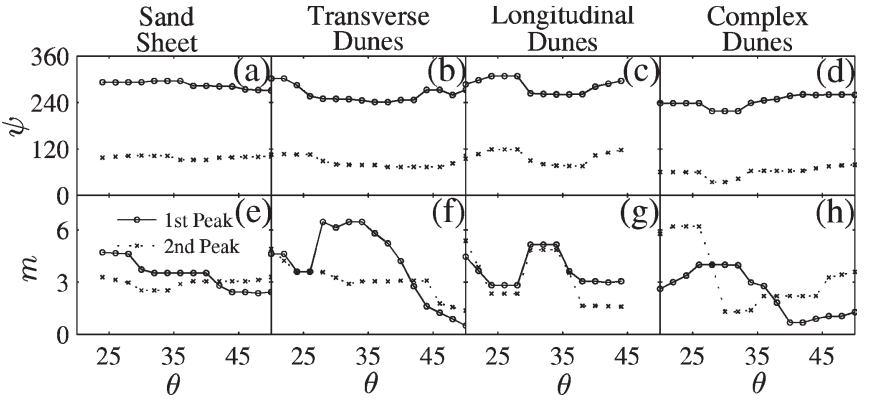

Fig. 10. (a)-(d) $\psi$ as a function of $\theta$ showing both $\psi_{1}$ and $\psi_{2}$ over the four sand surface types at locations $16,5,10$, and 18. (e)-(h) Similar plots of $m$ as a function of $\theta$ showing both $m_{1}$ and $m_{2}$.

ESCAT data over all the selected locations that are listed in Table I. The comparison of Table I columns 5 and 6 shows that estimated slip-side orientations reasonably match the ECMWF wind modes over the areas of simple dune bedforms. The estimates have higher deviation from ECMWF wind modes over the areas of compound and complex dunes. These results confirm that $q_{\theta}(\phi)$ at $\theta=33^{\circ}$ has a peak at $\psi$ approximately equal to the mean wind direction. In the next section we analyze the spatial maps of the model parameters and compare the results to the ECMWF winds.

\section{B. Spatial Behavior of Model Parameters}

In this section, the spatial behavior of the model parameters is considered. Since the presence of slip-sides is manifested in the $\phi$-modulation at incidence angle range $30^{\circ}-35^{\circ}$, we show the spatial maps at $\theta=33^{\circ}$.

Fig. 11 shows the spatial maps of parameters estimated from NSCAT data during January 1997. Fig. 11(a) is the map of NSCAT mean response $A_{\theta}$ showing that the ergs have a mean $\sigma^{\circ}$ response below $-20 \mathrm{~dB}$. Fig. 11(b) shows average wind behavior during January 1997 over the major ergs of the Sahara. ECMWF mean wind directions are shown on a $1^{\circ} \times 1^{\circ}$ resolution grid whereas model parameters are computed on a $0.5^{\circ} \times 0.5^{\circ}$ resolution grid. $m_{1}$ and $m_{2}$ have high magnitudes over ergs compared to the other parts of the Sahara Desert. Since the maps are plotted for $\theta=33^{\circ}$, these magnitudes reflect the relative strength of the signal caused by slip-sides in different ergs. Note that in almost all ergs, the difference in the directions $\psi_{1}$ and $\psi_{2}$ is approximately equal to $180^{\circ}$. Comparing Fig. 11(b) and (d) reveals that, in general, $\psi_{1}$ is similar to the average wind direction in the ergs. Most of the slip-sides in the northern ergs have their orientation eastwards, matching the direction of westerly trade winds, whereas the southern ergs match the easterly trade winds.

The Sahara Desert includes large mountain ranges called Hamadas that block and divert the near-surface winds, resulting in wind shadows. We note that ergs are large bowl-like basins formed in the intermountain regions. The ECMWF winds do not include mountain effects but the ergs do effect the intra-erg wind behavior. $\psi$ is computed based on the surface geometrical characteristics and thus reflects the near surface wind characteristics. The observed $\psi$ reveals the wind diversion caused by the mountains. This is reflected in the $\psi$ spatial variability between the wind inlets and outlets of the ergs. These results are further 

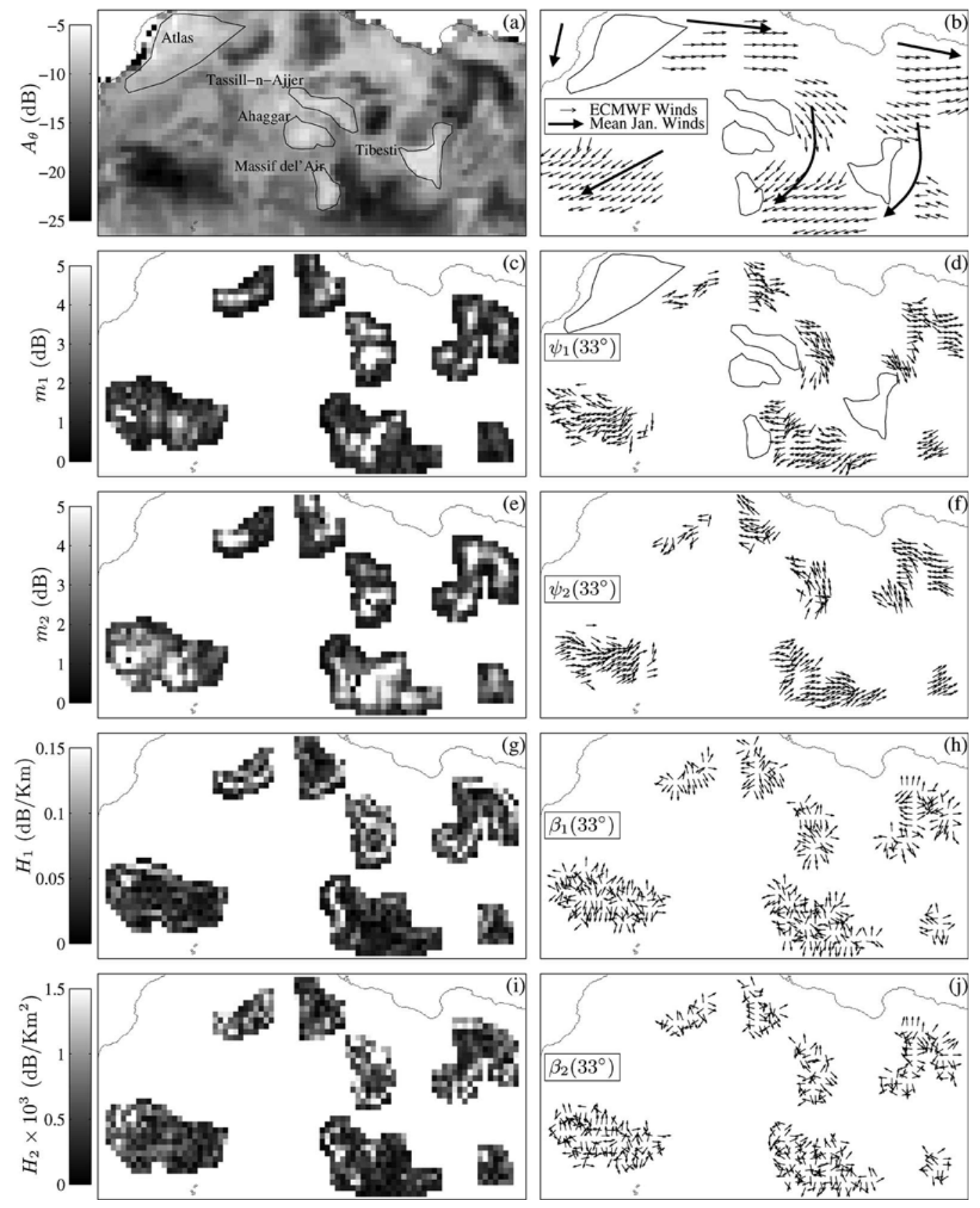

Fig. 11. Spatial maps of the model parameters at $\theta=33^{\circ}$ from NSCAT data during JD $1-30,1997$, for Saharan ergs. (a) $A_{\theta}$, (c) $m_{1}$, (d) $\psi_{1}$, (e) $m_{2}$, (f) $\psi_{2}$, (g) $H_{1}$, (h) $\beta_{1}$, (i) $H_{2}$, and (j) $\beta_{2}$. (b) Average wind behavior over the ergs during January (JD 1-30), 1997.

confirmed when compared to the average January wind maps reported by [16] and shown with thick arrows in Fig. 11(b). The rms deviation of $\psi_{1}$ to ECMWF mean winds is $27^{\circ}$.

The magnitude of the first-order spatial inhomogeneity is highest at the erg boundaries, consistent with a change of terrain from sandy surface to Hamadas. The high first-order spatial inhomogeneity magnitudes mark the boundaries of major ergs [see Fig. $11(\mathrm{~g})$ ]. In the central erg regions, $H_{1}$ is lower, indicating that the $\sigma^{\circ}$ is mainly effected by $q_{\theta}(\phi)$ and has a lesser contribution from $h_{\theta}(r, \beta)$. The second-order inhomogeneity is also high closer to the fringes of the ergs and its direction is almost equal to first-order inhomogeneity at most parts of the erg boundaries.
Fig. 12(a) and (b) compares the average July 1997 ECMWF winds to $\psi_{1}$ computed from ESCAT data and are similar to NSCAT. ESCAT $\psi_{1}$ has smoother spatial behavior because of the coarser ESCAT resolution and the fact that a $100-\mathrm{km}$ radius is used to compute the model parameters. The rms deviation of ESCAT $\psi_{1}$ from ECMWF winds is $29^{\circ}$. The first- and secondorder inhomogeneities are consistent with NSCAT. Fig. 12(c) is the spatial map of $\psi_{1}$ directions computed from QSCAT data. Average winds during July reported by [16] are also shown with thick arrows. We note that QSCAT $\psi_{1}$ is computed at $54^{\circ}$ and does not always reflect the orientation of the slip-side. This is evident by the higher deviations of its spatial map from the ECMWF winds. 


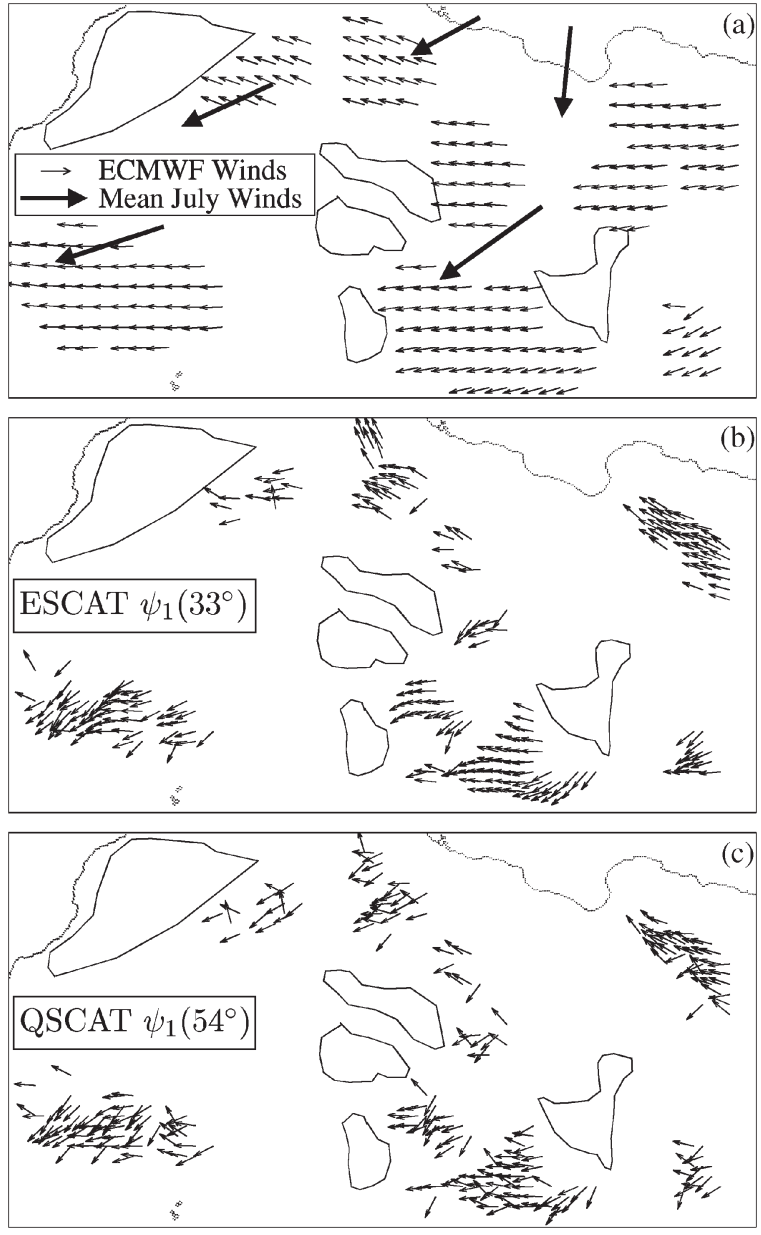

Fig. 12. (a) Average wind map JD 182-212, 1997, (b) $\psi_{1}$ computed for ESCAT data corresponding to (a), and (c) $\psi_{1}$ computed for QSCAT data during JD 182-212, 2000.

Fig. 13 shows a similar comparison in the empty quarter of the Arabian peninsula. NSCAT and ESCAT provide similar $\psi$ directions, which deviate from ECMWF winds. These $\psi$ directions are similar to the aeolian sand transport directions reported by [17], shown with dashed arrows in Fig. 13(a). Mainguet observed these sand transport paths in Landsat images identifying both transport-dominant and depositiondominant sectors [17]. The spatial behavior of $\psi$ is similar to the spatial behavior of the near-surface dominant wind directions. The model results have higher error in complex surface bedforms with more than two slip-sides. These bedforms result from highly variable wind directions and thus the surface bedform response to winds is more complicated compared to areas with simple dunes caused by less variable winds.

\section{Temporal Behavior of Model Parameters}

In this section, we compare the temporal variability of ECMWF average winds to the derived $\psi$ orientations. Fig. 14 shows plots of the monthly average ECMWF wind directions and $\psi$ orientations computed every 15 days between October 1996 and July 1997. In these locations, the ECMWF wind directions match reasonably well to the scatterometerderived $\psi$ orientations. The temporal variations of ECMWF
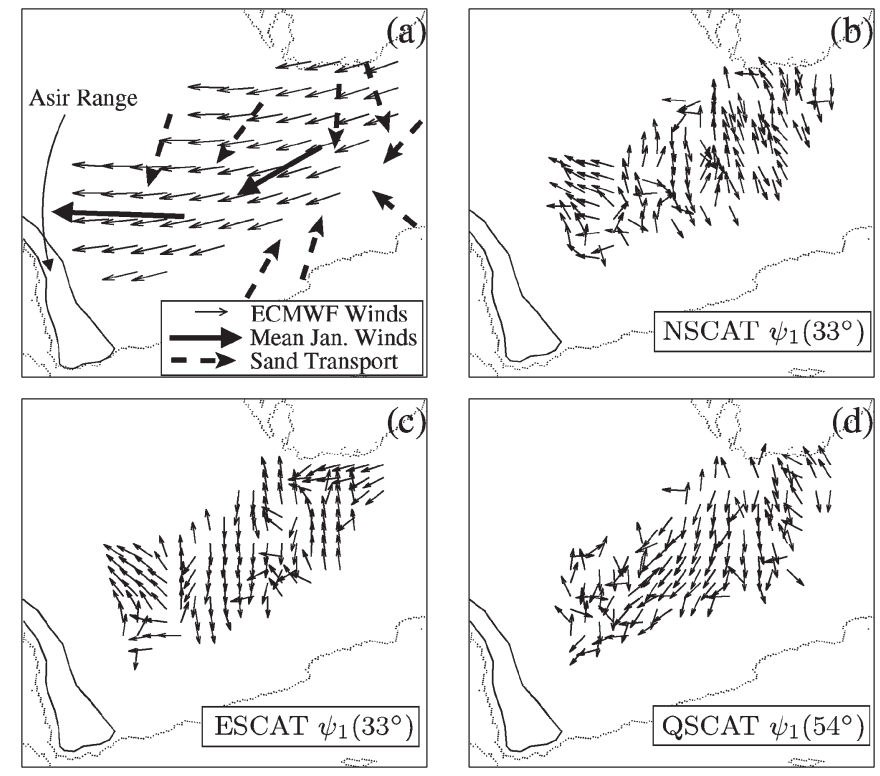

Fig. 13. (a) Average wind behavior in the empty quarter during JD 1-30, 1997. (b) and (c) $\psi$ spatial maps computed from NSCAT and ESCAT data during JD 1-30, 1997. (d) $\psi$ spatial map computed from QSCAT data during JD 1-30, 2000.

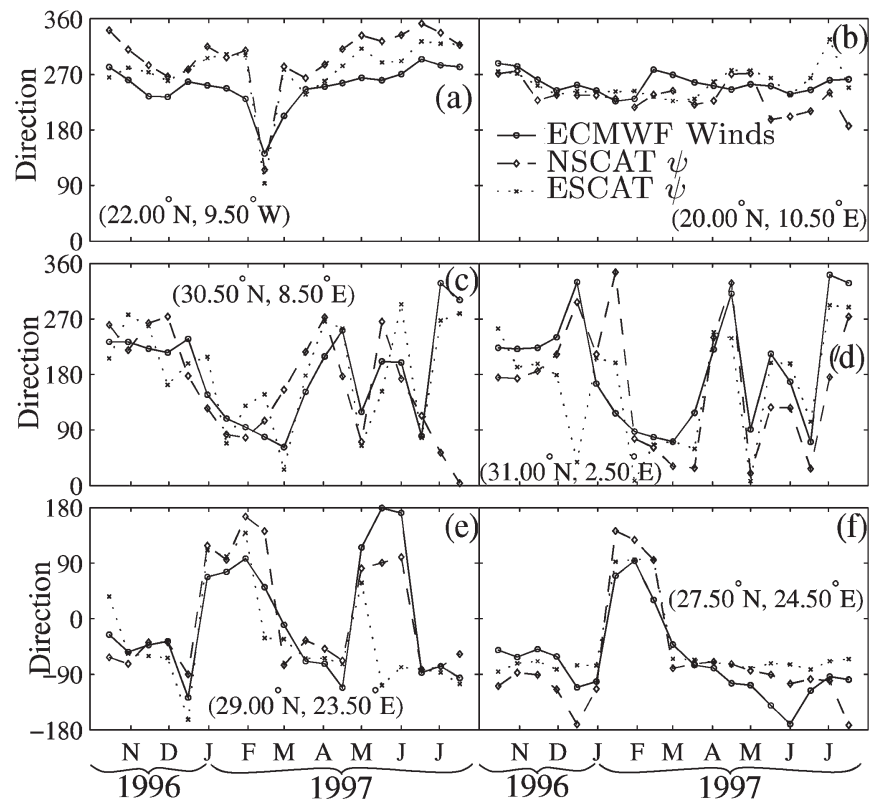

Fig. 14. Plots of temporal behavior ECMWF mean wind directions and $\psi$ orientations derived from NSCAT and ESCAT $\sigma^{\circ}$ measurements at six locations in Saharan ergs.

wind and $\psi$ are similar, but differ in absolute values. This suggests that the small-scale ripples are not absolutely aligned to the average prevailing winds but have a temporal behavior consistent with wind temporal variations in all the selected locations. The deviation of the two measurements increases as the surface complexity of the bedform increases. Fig. 15 shows the long-term comparison of ECMWF winds and $\psi$ computed from ESCAT at two locations.

The time series of $\psi$ and ECMWF wind direction are similar at many locations in the ergs. Where they differ, the difference is attributed to the different scopes of the two wind sources. 


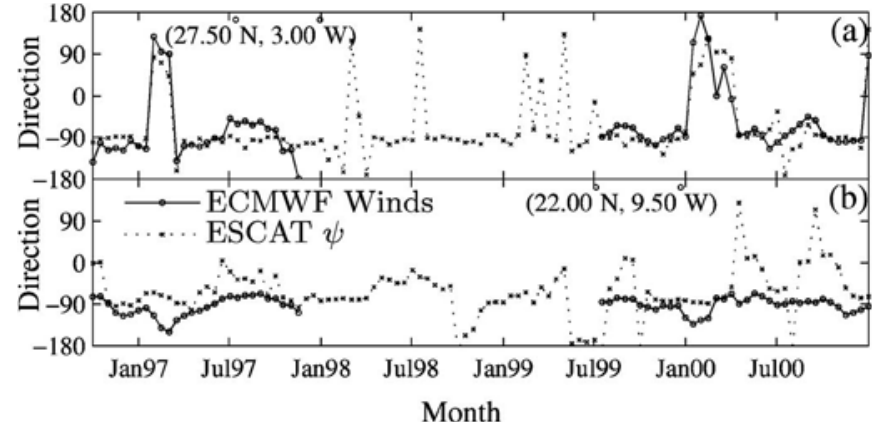

Fig. 15. Comparison of the long-term temporal behavior of ECMWF mean wind directions and $\psi$ orientations derived from ESCAT data at two locations.

ECMWF wind is numerically predicted and presents the largescale average behavior of the prevailing wind whereas $\psi$ is retrieved from the erg bedform geometry and is a measure of the near-surface aeolian phenomena.

\section{SUMMARY AND CONCLUSION}

The erg surface geometry response to the near-surface prevailing wind is very complicated. $\sigma^{\circ}$ measurements over ergs are influenced by the ergs' general topography and dune geometry, and are a function of sensor view angles and footprint centroid location. A new model includes both $\sigma^{\circ} \phi$-modulation and $\sigma^{\circ}$ spatial inhomogeneity and shows that, over ergs, $\theta$ plays a key role in varying the $\phi$-modulation and $\sigma^{\circ}$ centroid displacement variability. The $\phi$-modulation of $\sigma^{\circ}$ measurements at $\theta=33^{\circ}$ is used to relate the dune slip-side orientations to the prevailing wind over erg bedforms. Over the areas with simple dunes (one to two slip-sides) and less variable wind, the modelinferred wind is spatially and temporally similar to the ECMWF wind. NSCAT, ESCAT, and QSCAT show similar results. Since the model relies on the slip-side orientation, model accuracy reduces in the areas with higher spatial variability of slip-side orientations such as compound and complex dune bedforms. The model is also sensitive to the mixing of the backscatter from non-erg parts of desert in the measurements, especially at the erg boundaries.

\section{REFERENCES}

[1] R. U. Cooke and A. Warren, Geomorphology of Deserts. London, U.K.: B. T. Batsford Ltd., 1973.

[2] A. S. Goudie, I. Livingstone, and S. Stokes, Aeolian Environments, Sediments and Landforms. Chichester, U.K.: Wiley, 1999.

[3] K. Pye and H. Tsoar, Aeolian Sand and Sand Dunes. London, U.K.: Unwin Hyman Ltd., 1990.

[4] R. A. Bagnold, The Physics of Blown Sand and Desert Dunes. London, U.K.: Methuen, 1941.

[5] L. Bateson and I. H. Woodhouse, "Observations of scatterometer asymmetry over sand seas and derivation of wind ripple orientation," Int. J. Remote Sens., vol. 25, no. 10, pp. 1805-1816, May 2004.

[6] F. M. Naderi, M. H. Freilich, and D. G. Long, "Spaceborne radar measurement of wind velocity over the ocean-An overview of the NSCAT scatterometer system," Proc. IEEE, vol. 79, no. 6, pp. 850-866, Jun. 1991.

[7] E. P. W. Attema, "The Active Microwave Instrument on-board the ERS-1 satellite," Proc. IEEE, vol. 79, no. 6, pp. 791-799, Jun. 1991.

[8] M. Spencer, C. Wu, and D. G. Long, "Tradeoffs in the design of a spaceborne scanning pencil beam scatterometer: Application to SeaWinds," IEEE Trans. Geosci. Remote Sens., vol. 35, no. 1, pp. 115-126, Jan. 1997.
[9] H. Stephen and D. G. Long, "Analysis of scatterometer observations of Saharan ergs using a simple rough facet model," in Proc. IGARSS, Anchorage, AK, Sep. 20-24, 2004, vol. 3, pp. 1534-1537.

[10] H. Stephen and D. G. Long, "Microwave backscatter modeling of erg surfaces in the Sahara Desert," IEEE Trans. Geosci. Remote Sens., vol. 43, no. 2, pp. 238-247, Feb. 2005.

[11] H. Stephen and D. G. Long, "Modeling microwave emission of erg surfaces in the Sahara Desert," IEEE Trans. Geosci. Remote Sens., vol. 43, no. 12 , pp. 2822-2830, Dec. 2005.

[12] I. S. Ashcraft and D. G. Long, "Observation and characterization of radar backscatter over Greenland," IEEE Trans. Geosci. Remote Sens., vol. 43, no. 2, pp. 225-237, Feb. 2005.

[13] R. G. Kennett and F. K. Li, "Seasat over-land scatterometer data, Part I: Global overview of the ku-band backscatter coefficients," IEEE Trans. Geosci. Remote Sens., vol. 27, no. 5, pp. 592-605, Sep. 1989.

[14] H. Hersbach and S. Abdalla, "The global validation of ERS wind and wave product at ECMWF," in Proc. Envisat and ERS Symp., 2004, vol. ESA SP-572, pp. 432-437.

[15] E. D. McKee, A Study of Global Sand Seas: Geological Survey Professional Paper 1052. Washington DC: U.S. Gov. Printing Off., 1979.

[16] T. T. Warner, Desert Meteorology. Cambridge, U.K.: Cambridge Univ. Press, 2004.

[17] M. Mainguet, Space Observation of Saharan Aeolian Dynamics. Dordrecht, The Netherlands: Matinus Nijhoff, 1984.

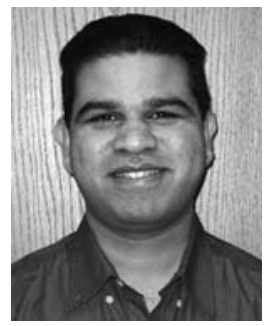

Haroon Stephen (S'97-M'07) received the Ph.D. degree in electrical and computer engineering from Brigham Young University (BYU), Provo, UT, in 2006.

During his Ph.D., he conducted research with the Microwave Earth Remote Sensing Laboratory and has also instructed undergraduate classes. He was with the European Space Agency office at the Asian Institute of Technology from 1997 to 1998, where he investigated scatterometer applications to study land surfaces. During this time, he also taught short courses in remote sensing. Currently, he is a Postdoctoral Research Fellow with the Wireless Communications Research Laboratory at BYU. His current research interests include optimization of wireless multiple-input multipleoutput (MIMO) systems, modeling of time-varying wireless MIMO channels, and microwave remote sensing of sand surfaces and tropical vegetation using spaceborne scatterometers and radiometers.

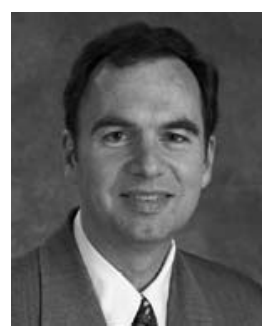

David G. Long (S'80-SM'98) received the Ph.D. degree in electrical engineering from the University of Southern California, Los Angeles, in 1989.

From 1983 to 1990 , he was with the National Aeronautics and Space Administration (NASA) Jet Propulsion Laboratory (JPL), Pasadena, where he developed advanced radar remote sensing systems. While at JPL, he was the Senior Project Engineer on the NASA Scatterometer project, which was flown aboard the Japanese Advanced Earth Observing System from 1996 to 1997. He was also the Experiment Manager and Project Engineer for the Scanned Scatterometer (now known as SeaWinds). In 1990, he joined the Department of Electrical and Computer Engineering, Brigham Young University (BYU), Provo, UT, where he currently teaches upper division and graduate courses in communications, microwave remote sensing, radar, and signal processing, is the Director of BYU's Center for Remote Sensing, and is the Head of the Microwave Earth Remote Sensing Laboratory. He is the Principle Investigator on several NASA-sponsored interdisciplinary research projects in remote sensing and innovative radar systems. He has numerous publications in signal processing and radar scatterometry. His research interests include microwave remote sensing, radar, polar ice, signal processing, estimation theory, and mesoscale atmospheric dynamics. He has over 280 publications in the open literature.

Dr. Long has received the NASA Certificate of Recognition several times. $\mathrm{He}$ is an Associate Editor for the IEEE Geoscience and Remote Sensing LETTERS. 